\title{
The Effects of Intensive Blood Pressure Control on Cardiovascular Outcomes Based on 10-Year ASCVD Risk Score: An Analysis of a Clinical Trial
}

\author{
Alireza Alborzi, ${ }^{1,2}$ Armin Attar $(D),{ }^{1}$ Mehrab Sayadi $\left(D,{ }^{1}\right.$ and Fatemeh Nouri ${ }^{2}$ \\ ${ }^{1}$ Department of Cardiovascular Medicine, Shiraz University of Medical Sciences, Shiraz, Iran \\ ${ }^{2}$ Student's Research Committee, Shiraz University of Medical Sciences, Shiraz, Iran \\ Correspondence should be addressed to Armin Attar; attar_armin@yahoo.com
}

Received 26 October 2020; Revised 15 April 2021; Accepted 3 May 2021; Published 12 May 2021

Academic Editor: Frank Dini

Copyright (C) 2021 Alireza Alborzi et al. This is an open access article distributed under the Creative Commons Attribution License, which permits unrestricted use, distribution, and reproduction in any medium, provided the original work is properly cited.

There is still controversy about whether clinicians should include cardiovascular disease (CVD) risk stratification into the consideration for treatment of hypertension. This was a post hoc analysis of the Systolic Blood Pressure Intervention Trial (SPRINT). A total of 9361 nondiabetic patients without a history of stroke were randomly assigned to the intensive-treatment group (with an SBP target of $<120 \mathrm{~mm} \mathrm{Hg}$ ) and the standard-treatment group (with an SBP target of $<140 \mathrm{~mm} \mathrm{Hg}$ ). The patients were categorized into four groups based on the Atherosclerotic Cardiovascular Disease (ASCVD) risk score. The groups contained participants with ASCVD $<7.5 \%, 7.5 \% \leq$ ASCVD $<10 \%, 10 \% \leq$ ASCVD $<15 \%$, and ASCVD $\geq 15 \%$. The incidence of the primary outcome, secondary outcome, and serious adverse events was compared between the two groups. The primary outcome was a composite of nonfatal myocardial infarction (MI), acute coronary syndrome (ACS) not resulting in MI, stroke, acute decompensated heart failure (HF), or death from cardiovascular causes. The secondary outcomes consisted of the individual components of the primary outcome and all-cause death. Intensive blood pressure (BP) control significantly reduced the incidence of primary outcome event in patients with $10 \% \leq$ ASCVD $<15 \%$ (hazard ratio (HR) $0.593 ; 95 \%$ confidence interval (CI) $0.361-0.975 ; P=0.039$ ) and ASCVD $\geq 15 \%$ (HR 0.778; CI 0.644-0.940; $P=0.009$ ). Intensive BP control was also beneficial for the primary prevention of cardiovascular events in patients with an ASCVD risk of $7.5-10 \%$ (HR 0.187; 95\% CI 0.040-0.862; $P=0.032$ ). However, intensive treatment was associated with higher incidence of hypotension and acute renal failure in participants with ASCVD $\geq 15 \%$. In patients without diabetes mellitus and prior stroke who had a 10-year risk of cardiovascular events above $10 \%$ based on the ASCVD risk score, intensive BP control played an important role in the reduction of major cardiovascular events. Additionally, intensive treatment would be beneficial for primary prevention in patients with ASCVD $\geq 7.5 \%$ without previous history of any cardiovascular disorders. Trial registration: ClinicalTrials.gov number; the trial is registered with NCT01206062.

\section{Introduction}

Hypertension is a prevalent chronic disease, especially in the elderly population, that leads to stroke, end-stage renal disease (ESRD), myocardial infarction (MI), congestive heart failure (CHF), and peripheral vascular disease [1].

Adequate control of hypertension plays a crucial role in cardiovascular disease (CVD) rate and subsequent mortality reduction and is much more cost-effective than treating cardiovascular events that result from uncontrolled hypertension [2]. Systolic blood pressure (SBP) is a more important predictor of cardiovascular events compared with diastolic blood pressure (DBP) [3]. It has been shown that antihypertensive treatment for an SBP of $140 \mathrm{mmHg}$ or higher is associated with a reduced risk of death and CVD, and treatment is recommended for these patients [4]. However, some studies have suggested that intensive blood pressure (BP) reduction to a target $\mathrm{SBP} \leq 120 \mathrm{~mm} \mathrm{Hg}$ should be considered to decrease the risk of cardiovascular events in some special people $[5,6]$. 
The Systolic Blood Pressure Intervention Trial [5] was a multicenter trial of 9361 adults $\geq 50$ years of age, with a systolic blood pressures of $\geq 130 \mathrm{~mm} \mathrm{Hg}$ and at least one additional cardiovascular risk factor, who were randomly assigned to either intensive (SBP target: $\leq 120 \mathrm{~mm} \mathrm{Hg}$ ) or standard (SBP target: $\leq 140 \mathrm{~mm} \mathrm{Hg}$ ) treatment group [7]. It was concluded that, in the patients with high CVD risk, targeting an SBP less than $120 \mathrm{~mm} \mathrm{Hg}$ as compared with an SBP less than $140 \mathrm{~mm} \mathrm{Hg}$ resulted in lower rates of fatal and nonfatal major cardiovascular events and death from any cause [5].

Clinical practice guidelines for hypertension treatment relied primarily on the BP levels. However, several studies have provided support for the positive role of CVD risk assessment in guiding BP-lowering treatment decisions $[8,9]$. Several risk prediction tools have been developed to identify the patients at high risk of CVD, such as the Framingham Risk Score (FRS) and the Atherosclerotic Cardiovascular Disease (ASCVD) risk score. The ASCVD risk score is a formula developed by the American College of Cardiology and the American Heart Association for evaluation of the risk of future cardiovascular events [10].

In this study, we aimed to conduct a secondary analysis of SPRINT data in order to compare the effects of intensive BP control with an SBP target of less than $120 \mathrm{mmHg}$ and standard BP control with an SBP target of less than $140 \mathrm{mmHg}$ on cardiovascular outcomes in the patients who had different ASCVD risk scores at baseline.

\section{Methods}

2.1. Study Design and Population. This study was a post hoc analysis of SPRINT. The SPRINT data were obtained from the National Heart, Lung, and Blood Institute (NHLBI), Biologic Specimen and Data Repository Information Coordinating Center.

SPRINT was a randomized, controlled trial that was conducted at 102 clinical sites. In this study, a total of 9361 participants were enrolled. The patients were randomized into the intensive-treatment group with a target SBP of less than $120 \mathrm{mmHg}$ and the standard-treatment group targeting an SBP of less than $140 \mathrm{mmHg}$ (Figure 1) [5].

To be eligible for participation in the study, the patients were required to meet all the following criteria: (1) age $\geq 50$ years; (2) an SBP of 130-180 mm Hg; and (3) an increased risk of cardiovascular events, which was defined by one or more of the following criteria: clinical or subclinical CVD other than stroke; chronic kidney disease (CKD), excluding polycystic kidney disease, with an estimated glomerular filtration rate (eGFR) of 20 to less than $60 \mathrm{ml} / \mathrm{min} / 1.73 \mathrm{~m}^{2}$; a 10 -year risk of $\mathrm{CVD} \geq 15 \%$ on the basis of FRS; or age $\geq 75$ years. The main exclusion criteria were one-minute standing SBP $<110 \mathrm{~mm}$ $\mathrm{Hg}$, proteinuria $\geq 1 \mathrm{~g} /$ day, diabetes mellitus (DM), history of stroke, polycystic kidney disease, eGFR $<20 \mathrm{ml} / \mathrm{min} / 1.73 \mathrm{~m}^{2}$ or end-stage renal disease (ESRD), symptomatic heart failure (HF) within the past 6 months, and pregnancy [5].

The ASCVD risk score is a continuous score, ranging from $0 \%$ to $100 \%$, to estimate the risk of cardiovascular events in the next 10 years on the basis of variables, including SBP, total cholesterol (TC), high-density lipoprotein cholesterol (HDLC), age, sex, race, diabetes, and smoking status [11]. According to the ASCVD risk score, we divided the participants of SPRINT into four groups: ASCVD $<7.5 \%, \quad 7.5 \% \leq$ ASCVD $<10 \%, \quad 10 \% \leq \mathrm{ASCVD}<15 \%$, and ASCVD $\geq 15 \%$. This study has been approved by the national ethical committee by an approval number of IR.SUMS.MED.REC.1398.377 and conforms to the Declaration of Helsinki. All participants signed the consent form.

2.2. Intervention and Measurements. Participants were randomly assigned to an SBP target of either $<140 \mathrm{~mm} \mathrm{Hg}$ (the standard-treatment group) or $<120 \mathrm{~mm} \mathrm{Hg}$ (the intensive-treatment group). All major classes of antihypertensive drugs were included in the formulary. For participants in the intensive-treatment group, medications were adjusted to target an SBP $<120 \mathrm{~mm} \mathrm{Hg}$. Medications for participants in the standard-treatment group were adjusted to target an SBP of 135-139 $\mathrm{mm} \mathrm{Hg}$, and the dose decreased if SBP was $<130 \mathrm{~mm} \mathrm{Hg}$ on a single visit or $<135 \mathrm{~mm} \mathrm{Hg}$ on two consecutive visits [5].

The mean of three BP measurements at an office visit while the patient was seated and after five minutes of quiet rest was considered as a basis for dose adjustment of drugs. Blood pressure measurements were done by an automated measurement system (Model 907, Omron Healthcare) [5].

Demographic data were obtained from participants at baseline. Clinical and laboratory data were recorded at baseline and then every 3 months. In addition, a structured interview was done every 3 months to obtain self-reported CVD outcomes [5].

2.3. Clinical Outcomes. The primary end point was a composite of nonfatal MI, acute coronary syndrome (ACS) not resulting in $\mathrm{MI}$, stroke, acute decompensated $\mathrm{HF}$, or death from cardiovascular causes [12]. The secondary outcomes were the individual components of the primary outcome and all-cause death [13].

2.4. Serious Adverse Events. Serious adverse events (SAEs) were defined as events that met any of the following criteria: (1) being fatal or life-threatening; (2) resulting in significant or persistent disability; (3) requiring or prolonged hospitalization; or (4) suffering important medical event that is judged to represent significant hazards to participants and may require medical or surgical intervention. Adverse events consisted of syncope, bradycardia, hypotension, electrolyte disturbances, and acute renal failure (AKI) which were evaluated at the emergency department [14].

2.5. Statistical Analysis. Statistical analysis was performed using the chi-square test, the one-way ANOVA test, and the proportional hazard cox regression model. A $P$ value less than 0.05 was considered significant. SPSS version 22 (IBM SPSS, Armonk, NY, USA) was used to perform data analysis. 


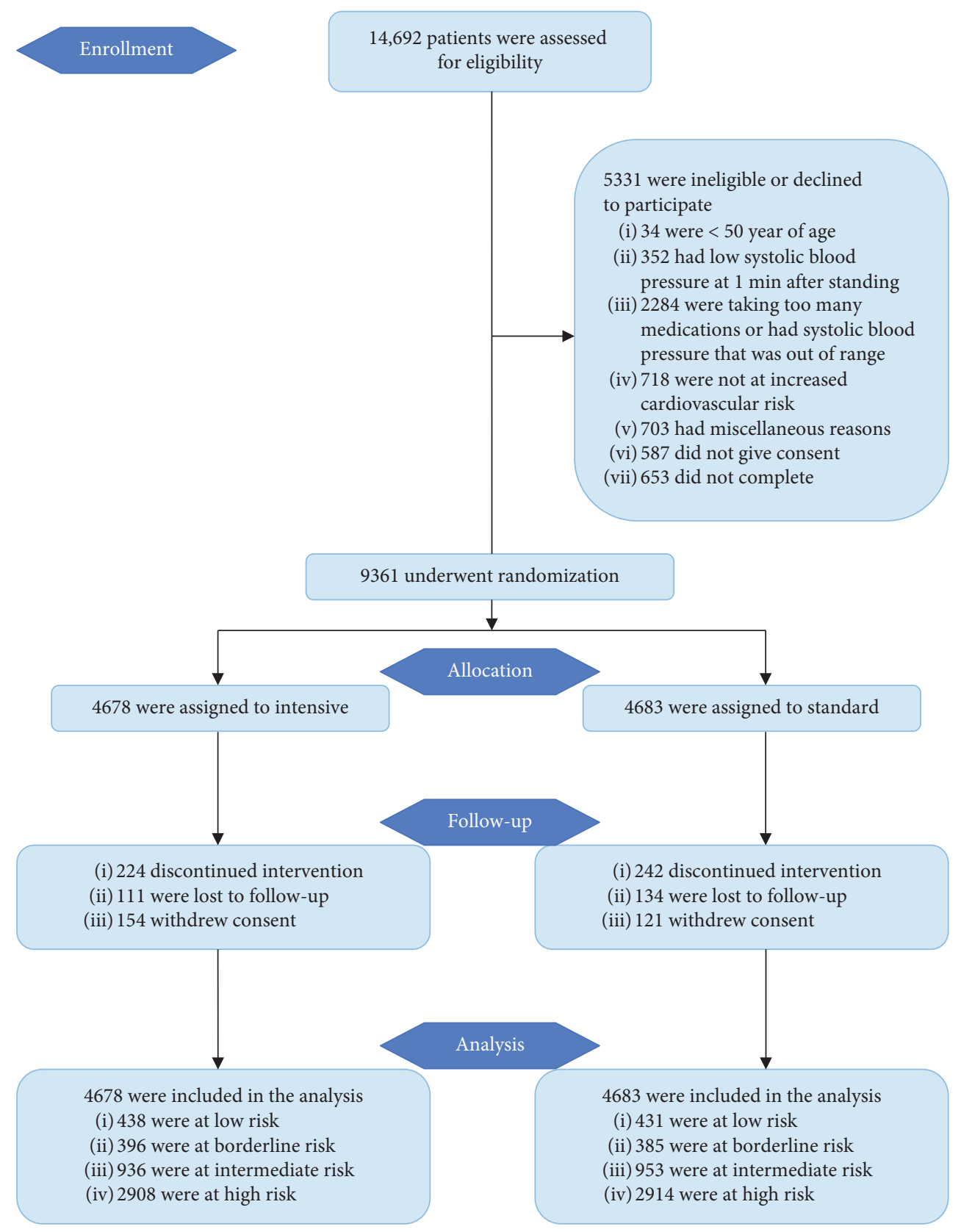

Figure 1: CONSORT flow diagram.

\section{Results}

3.1. Study Participants. We used data of 9361 patients who participated in the SPRINT trial. There were $869,781,1889$, and 5822 patients with ASCVD $<7.5 \%, 7.5 \% \leq$ ASCVD $<10 \%$, $10 \% \leq \mathrm{ASCVD}<15 \%$, and $\mathrm{ASCVD} \geq 15 \%$, respectively. The participants with ASCVD $\geq 15 \%$ were older and had higher SBP, triglycerides (TG), and glucose levels. Regarding various parameters, no statistically significant differences were found between the intensive- and standard-treatment group in each ASCVD category. The demographic and clinical data of the cases are summarized in Table 1.
3.2. Clinical Outcomes. A primary outcome event was reported in 22 patients with ASCVD $<7.5 \%-12$ in the standard-treatment group and 10 in the intensive-treatment group (hazard ratio (HR) with intensive treatment 0.668; 95\% confidence interval (CI) $0.272-1.637 ; P=0.378$ ); 29 patients with $7.5 \% \leq$ ASCVD $<10 \%-16$ in the standardtreatment group and 13 in the intensive-treatment group (HR 0.659; 95\% CI 0.294-1.478; $P=0.311$ ); 71 patients with $10 \% \leq \mathrm{ASCVD}<15 \%-45$ in the standard-treatment group and 26 in the intensive-treatment group (HR 0.593; 95\% CI $0.361-0.975 ; \quad P=0.039) ; \quad$ and 440 patients with ASCVD $\geq 15 \%-246$ in the standard-treatment group and 
TABLE 1: Demographic and clinical characteristics of participants with different baseline ASCVD risk scores.

\begin{tabular}{|c|c|c|c|c|c|c|c|c|}
\hline \multirow[t]{2}{*}{ Characteristics } & \multicolumn{2}{|c|}{ ASCVD below 7.5\% } & \multicolumn{2}{|c|}{$\begin{array}{c}\text { ASCVD equal to or } \\
\text { greater than } 7.5 \% \text { and less } \\
\text { than } 10 \%\end{array}$} & \multicolumn{2}{|c|}{$\begin{array}{l}\text { ASCVD equal to or greater } \\
\text { than } 10 \% \text { and less than } 15 \%\end{array}$} & \multicolumn{2}{|c|}{$\begin{array}{c}\text { ASCVD equal to or greater } \\
\text { than } 15 \% .\end{array}$} \\
\hline & Intensive & Standard & Intensive & Standard & Intensive & Standard & Intensive & Standard \\
\hline $\mathrm{N}$ & $438(9.4)$ & $431(9.2)$ & $396(8.5)$ & $385(8.2)$ & $936(20.0)$ & $953(20.4)$ & $2908(62.2)$ & $2914(62.2)$ \\
\hline ASCVD\% & $5.44 \pm 1.43$ & $5.41 \pm 1.53$ & $8.84 \pm 0.73$ & $8.73 \pm 0.73$ & $12.49 \pm 1.45$ & $12.48 \pm 1.41$ & $30.16 \pm 13.75$ & $30.07 \pm 13.59$ \\
\hline Age, y & $57.35 \pm 4.24$ & $57.07 \pm 4.42$ & $60.26 \pm 4.80$ & $59.81 \pm 5.02$ & $62.25 \pm 5.68$ & $62.35 \pm 5.75$ & $72.39 \pm 8.35$ & $72.40 \pm 8.40$ \\
\hline Female & $298(17.7)$ & $288(17.5)$ & $207(12.3)$ & $194(11.8)$ & $345(20.5)$ & $337(20.4)$ & $834(49.5)$ & $829(50.3)$ \\
\hline \multicolumn{9}{|l|}{ Smoking status } \\
\hline Never & $237(13.3)$ & $252(12.2)$ & $230(9.9)$ & $199(9.6)$ & $435(21.2)$ & $443(21.4)$ & $1139(55.6)$ & $1178(56.9)$ \\
\hline Former & $134(6.8)$ & $149(7.5)$ & $154(7.8)$ & $148(7.4)$ & 377 (19.1) & & $1312(66.4)$ & $1302(65.2)$ \\
\hline Current & $31(4.9)$ & $30(5.0)$ & $39(6.1)$ & $38(6.3)$ & $123(19.2)$ & $112(18.6)$ & $867(69.9)$ & $446(69.8)$ \\
\hline $\mathrm{SBP}, \mathrm{mmHg}$ & $131.1 \pm 14.5$ & $129.9 \pm 14.1$ & $133.3 \pm 13.6$ & $133.6 \pm 13.3$ & $136.5 \pm 14.7$ & $136.7 \pm 1$ & $142.8 \pm 15.5$ & $142.8 \pm 15.2$ \\
\hline $\mathrm{DBP}, \mathrm{mmHg}$ & $80.6 \pm 10.7$ & $80.0 \pm 10.9$ & $80.2 \pm 11.0$ & $80.3 \pm 10.3$ & $80.5 \pm 11.0$ & $80.5 \pm 11.3$ & $76.8 \pm 12.2$ & $78.0 \pm 12.3$ \\
\hline BMI, $\mathrm{kg} / \mathrm{m}^{2}$ & $31.9 \pm 6.7$ & $31.9 \pm 6.4$ & $31.5 \pm 6.2$ & $31.4 \pm 6.8$ & $30.9 \pm 6.2$ & $30.9 \pm 5.7$ & $29.0 \pm 5.2$ & $28.8 \pm 5.2$ \\
\hline $\mathrm{TC}, \mathrm{mg} / \mathrm{dL}$ & $195.5 \pm 42.7$ & $194.7 \pm 44.3$ & $194.7 \pm 41.1$ & $192.8 \pm 37.7$ & $191.8 \pm 40.7$ & $190.4 \pm 40.3$ & $188.2 \pm 41.3$ & $188.8 \pm 40.9$ \\
\hline $\mathrm{HDL}, \mathrm{mg} / \mathrm{dL}$ & $54.7 \pm 15.3$ & $55.0 \pm 14.9$ & $53.2 \pm 15.1$ & $53.2 \pm 14.4$ & $52.1 \pm 12.9$ & $51.9 \pm 14.0$ & $52.9 \pm 14.4$ & $52.7 \pm 14.7$ \\
\hline $\mathrm{TG}, \mathrm{mg} / \mathrm{dL}$ & $3.8 \pm 64.9$ & $125.9 \pm 79.4$ & $124.4 \pm 66.0$ & $119.4 \pm 67.6$ & $124.0 \pm 102.0$ & $127.9 \pm 8$ & $125.2 \pm 85.1$ & $128.0 \pm 102.2$ \\
\hline Glucose, mg/dL & $96.9 \pm 10.8$ & $97.4 \pm 10.6$ & $98.3 \pm 11.7$ & $96.7 \pm 11.4$ & $97.8 \pm 12.6$ & $98.2 \pm 11.6$ & \pm 14.6 & $99.4 \pm 14.4$ \\
\hline ALCR, mg/g & $24.9 \pm 84.1$ & $35.1 \pm 140.9$ & $24.5 \pm 75.6$ & $32.3 \pm 148.3$ & $35.8 \pm 177.9$ & $39.3 \pm 183.2$ & $52.2 \pm 197.7$ & $43.7 \pm 144.0$ \\
\hline EGFR & $75.6 \pm 20.5$ & $75.1 \pm 21.6$ & $75.3 \pm 22.0$ & $77.4 \pm 21.0$ & $76.6 \pm 20.9$ & $74.8 \pm 19.7$ & $69.0 \pm 19.9$ & $69.4 \pm 20.1$ \\
\hline CKD history & $90(6.8)$ & & $91(6.8)$ & & $185(13.9)$ & & $968(72.5)$ & $951(72.3)$ \\
\hline CVD history & $80(8.5)$ & $79(5.4)$ & & $63(6.7)$ & $140(14.9)$ & $175(18.7)$ & $656(69.8)$ & $620(66.2)$ \\
\hline \multicolumn{9}{|l|}{ N_AGENTS } \\
\hline 0 & $68(15.7)$ & $46(10.0)$ & $53(12.3)$ & $52(11.6)$ & $111(25.7)$ & $121(26.9)$ & $200(46.3)$ & $232(51.6)$ \\
\hline 1 & $136(10.0)$ & $124(8.9)$ & $118(8.6)$ & $113(8.1)$ & $254(18.6)$ & $263(18.9)$ & $857(62.8)$ & $888(64.0)$ \\
\hline 2 & $131(7.9)$ & $168(10.3)$ & $140(8.4)$ & $127(7.8)$ & $322(19.3)$ & 307 (18.9) & $1072(64.4)$ & $1025(63.0)$ \\
\hline 3 & $79(8.3) \mathrm{T}$ & $77(8.0)$ & $70(7.3)$ & $74(7.7)$ & $195(20.4)$ & $196(20.3)$ & $612(64.0)$ & $617(64.0)$ \\
\hline 4 & $23(9.1)$ & $17(7.0)$ & $15(5.9)$ & $17(7.0)$ & $54(21.3)$ & $65(26.6)$ & $161(63.6)$ & $154(59.4)$ \\
\hline 5 & $1(16.7)$ & 0 & 0 & $2(20)$ & 0 & $1(10)$ & $5(83.3)$ & $7(70.0)$ \\
\hline 6 & 0 & 0 & 0 & 0 & 0 & 0 & $1(100)$ & 0 \\
\hline Statin & $153(7.7)$ & $167(8.0)$ & $147(7.4)$ & $134(6.5)$ & $357(18.0)$ & $400(19.3)$ & $1321(66.8)$ & $1375(66.2)$ \\
\hline
\end{tabular}

Data were presented as mean \pm SD or number (\%) for continuous and categorical variables, respectively. ASCVD: Atherosclerotic Cardiovascular Disease, SBP: systolic blood pressure, DBP: diastolic blood pressure, BMI: body mass index, TC: total cholesterol, HDL: high density lipoprotein, TG: triglycerides, ALCR: albumin-to-creatinine ratio, EGFR: estimated glomerular filtration rate, CKD: chronic kidney disease, CVD: cardiovascular disease, N_AGENTS: number of antihypertensive agents.

194 in the intensive-treatment group (HR 0.778; 95\% CI $0.644-0.940 ; P=0.009)$. Generally, intensive BP control had a significant role in the reduction of the primary outcome in the patients with $A S C V D \geq 10$ (Table 2, Figure 2). Intensive $\mathrm{BP}$ control also reduced the incidence of HF (HR 0.61; 95\% CI $0.44-0.84 ; P=0.003$ ) and CVD (HR 0.56; 95\% CI $0.37-0.84 ; P=0.006)$ in the total population regardless of ASCVD risk scores. The details of secondary outcomes are shown in Table 3. Additionally, in terms of primary prevention (those without previous cardiovascular disease), intensive treatment was beneficial in participants with ASCVD $\geq 7$ 5\% (HR 0.187; 95\% CI 0.040-0.862; $P=0.032$ ).

3.3. Serious Adverse Events. SAEs consisted of hypotension, syncope, bradycardia, AKI, and electrolyte abnormality. Intensive treatment-related SAEs showed an increased risk of hypotension and syncope among the participants with ASCVD $\geq 15 \%$ and $10 \% \leq$ ASCVD $<15 \%$, respectively. The incidence of AKI was significantly higher in the patients with $7.5 \% \leq \mathrm{ASCVD}<10 \%$ and ASCVD $\geq 15 \%$. Additionally, in the total population, the risk of hypotension, AKI, and electrolyte abnormality significantly increased. ASCVDstratified subgroup analysis of SAEs between intensive- and standard-treatment group is detailed in Table 4.

3.4. Patients at High ASCVD Risk. We did perform an analysis in the subpopulation of patients with an ASCVD risk $\geq 15$ since they represent $62 \%$ of the total study population. Among them, 440 patients, 246 in the standardtreatment group and 194 in the intensive-treatment group (HR 0.778; 95\% CI 0.644-0.940; $P=0.009$ ), developed with a primary outcome. In addition, we performed a retrospective comparison of baseline characteristics between patients who had developed AKI and those who did not in the whole study population; those who had developed AKI had a lower eGFR (56.28\% vs. $72.50 \%, P<.001)$ and a higher serum creatinine level $(1.43$ vs. $1.05, P<.001)$ compared to those who did not develop AKI. In fact, the presence of CKD increased the chance of AKI by $215 \%$, and an eGFR below $62.04 \%$ predicted the occurrence of AKI with $66.77 \%$ sensitivity and 69.39\% specificity (area under the curve, 0.715; $P<.001$, Figure 3). 
TABLE 2: Primary outcome in the intensive- and standard-treatment group.

\begin{tabular}{|c|c|c|c|}
\hline \multirow{2}{*}{$\frac{\text { Subgroups }}{\text { ASCVD }<7.5 \%}$} & \multicolumn{3}{|c|}{ Primary outcome } \\
\hline & \multicolumn{2}{|c|}{ Number } & \multirow{3}{*}{ Reference } \\
\hline \multirow{2}{*}{ Standard } & Yes & 12 & \\
\hline & No & 419 & \\
\hline \multirow{2}{*}{ Intensive } & Yes & 10 & \multirow{2}{*}{$0.668(0.272-1.637), 0.378$} \\
\hline & No & 428 & \\
\hline \multicolumn{4}{|c|}{$7.5 \% \leq \mathrm{ASCVD}<10 \%$} \\
\hline \multirow{2}{*}{ Standard } & Yes & 16 & \multirow{2}{*}{ Reference } \\
\hline & No & 369 & \\
\hline \multirow{2}{*}{ Intensive } & Yes & 13 & \multirow{2}{*}{0.659 (0.294-1.478), 0.311} \\
\hline & No & 383 & \\
\hline \multicolumn{4}{|c|}{$10 \% \leq \mathrm{ASCVD}<15 \%$} \\
\hline \multirow{2}{*}{ Standard } & Yes & 45 & \multirow{2}{*}{ Reference } \\
\hline & No & 908 & \\
\hline \multirow{2}{*}{ Intensive } & Yes & 26 & \multirow{2}{*}{$0.593(0.361-0.975), \mathbf{0 . 0 3 9}$} \\
\hline & No & 910 & \\
\hline \multicolumn{4}{|l|}{$A S C V D \geq 15 \%$} \\
\hline \multirow{2}{*}{ Standard } & Yes & 246 & \multirow{2}{*}{ Reference } \\
\hline & No & 2668 & \\
\hline \multirow{2}{*}{ Intensive } & Yes & 194 & \multirow{2}{*}{$0.778(0.644-0.940), \mathbf{0 . 0 0 9}$} \\
\hline & No & 2714 & \\
\hline
\end{tabular}

Data were presented as hazard ratio (95\% confidence interval), $P$ value. The bold $P$ value data indicates its significance. ASCVD: Atherosclerotic Cardiovascular Disease.

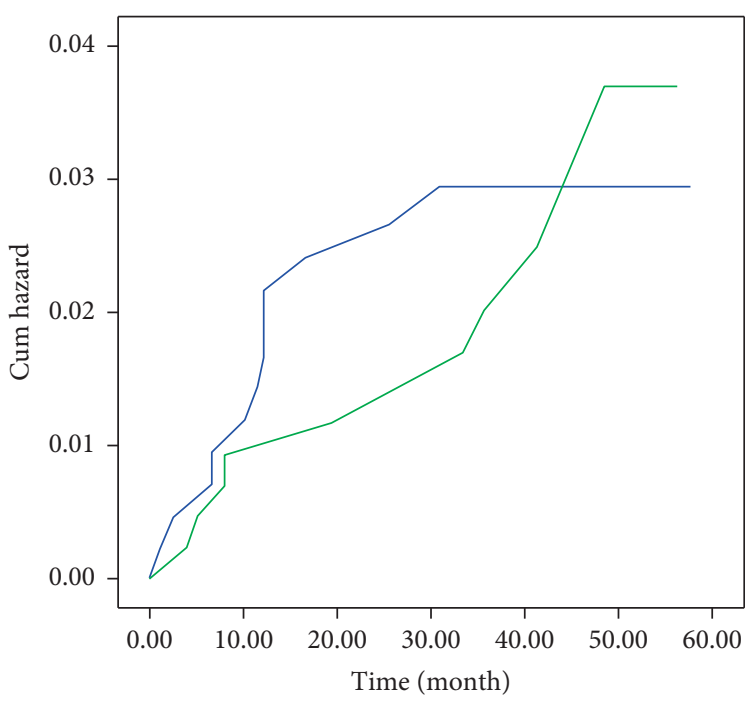

Treatment

- Standard BP arm

Intensive BP arm

(a)

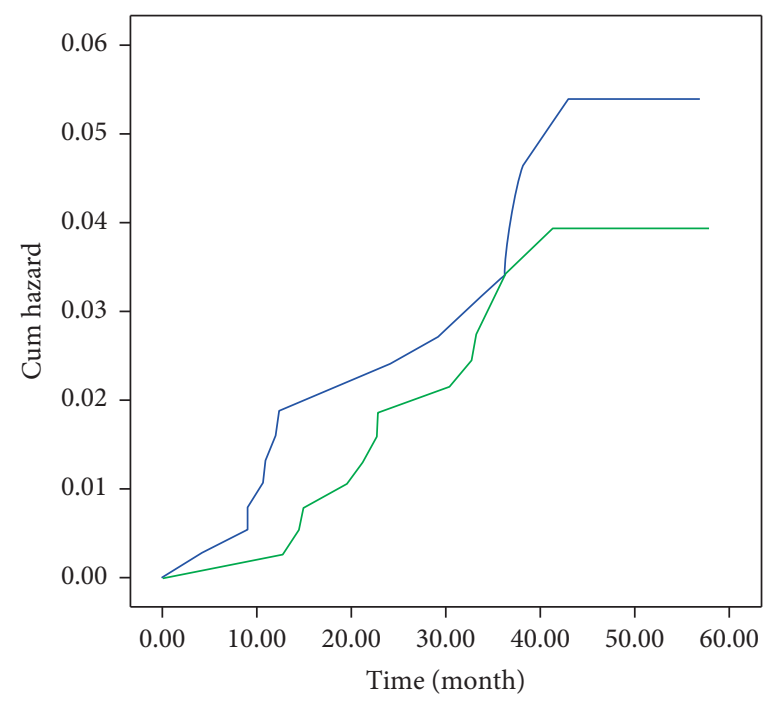

Treatment

- Standard BP arm

_ Intensive BP arm

(b)

Figure 2: Continued. 


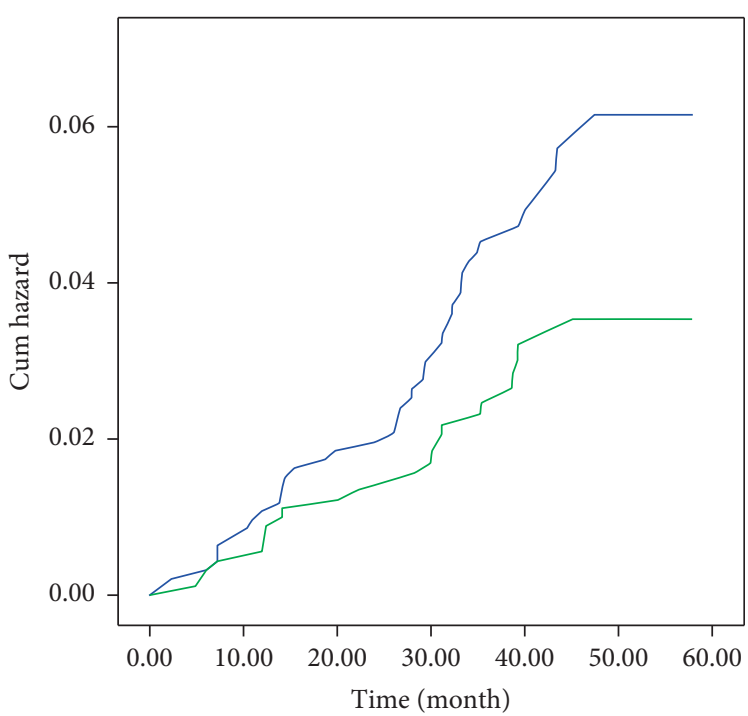

Treatment

- Standard BP arm

— Intensive BP arm

(c)

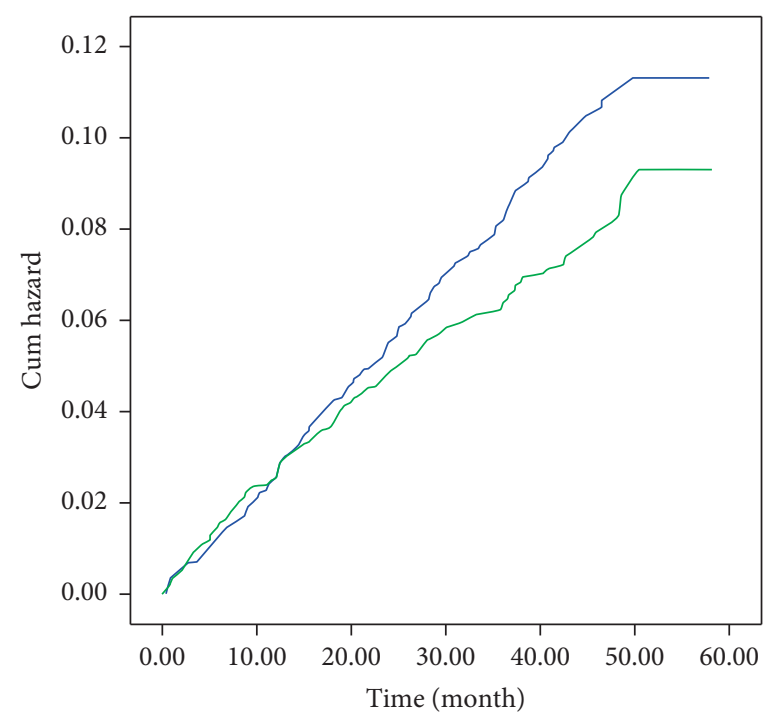

Treatment

- Standard BP arm

_ Intensive BP arm

(d)

Figure 2: ASCVD-stratified Kaplan-Meier curve analysis was performed to compare the primary outcome between the intensive-treatment group and the standard-treatment group among the patients with ASCVD $<7.5 \%, 7.5 \% \leq \mathrm{ASCVD}<10 \%, 10 \% \leq \mathrm{ASCVD}<15 \%$, and ASCVD $\geq 15 \%$. (a) Hazard function for patterns 1-2 ASCVD groups: ASCVD < 7.5. (b) Hazard function for patterns 1-2 ASCVD groups: $7.5 \leq$ ASCVD $<10$. (c) Hazard function for patterns 1-2 ASCVD groups: $10 \leq$ ASCVD $<15$. (d) Hazard function for patterns 1-2 ASCVD groups: $A S C V D \geq 15$.

TABLE 3: Secondary outcomes in the intensive- and standard-treatment group.

\begin{tabular}{lcccccc}
\hline \multirow{2}{*}{ Group } & ASCVD & \multicolumn{3}{c}{ Secondary outcomes } \\
& $\%$ & MI & Non-MI ACS & Stroke & HF & CVD death \\
\hline & $<7.5 \%$ & $0.39(0.07-2.01)$, & $0.01(0.00-1295)$, & $3.84(0.42-34.36)$, & $0.95(0.23-3.82)$, & $0.96(0.13-6.81)$, \\
& $7.5 \%-$ & $0.95(0.27-3.30)$, & $1.45(0.24-8.10)$, & $0.24(0.02-2.14)$, & $1.87(0.17-20.68)$, & $0.47(0.11-1.90)$, \\
& $10 \%$ & 0.945 & 0.681 & 0.202 & 0.608 & 0.296 \\
Intensive/ & $10 \%-$ & $0.48(0.20-1.11)$, & $0.58(0.17-1.98)$, & $1.79(0.52-6.12)$, & $0.51(0.21-1.19)$, & $0.53(0.14-1.47)$, \\
standard & $15 \%$ & 0.087 & 0.386 & 0.341 & 0.123 & 0.188 \\
& & $0.92(0.68-1.24)$, & $1.13(0.69-1.87)$, & $0.81(0.56-1.18)$, & $0.60(0.42-0.86)$, & $0.58(0.36-0.93)$, \\
& & 0.585 & 0.611 & 0.292 & $\mathbf{0 . 0 0 6}$ & $\mathbf{0 . 0 2 4}$ \\
& Total & $0.83(0.63-1.09)$, & $0.99(0.64-0.154)$, & $0.88(0.62-1.24), 0.472$ & $0.61(0.44-0.84)$, & $0.56(0.37-0.84)$, \\
& & 0.183 & 0.994 & $\mathbf{0 . 0 0 3}$ & $\mathbf{0 . 0 0 6}$ \\
\hline
\end{tabular}

Data were presented as hazard ratio (95\% confidence interval), $P$ value. The bold $P$ value data indicates its significance. ASCVD: Atherosclerotic Cardiovascular Disease, MI: myocardial infarction, ACS: acute coronary syndrome, HF: heart failure, CVD: cardiovascular disease.

TABLE 4: Serious adverse events in the intensive- and standard-treatment group.

\begin{tabular}{|c|c|c|c|c|c|}
\hline ASCVD & Hypotension & Syncope & Bradycardia & Acute renal failure & Electrolyte abnormality \\
\hline $\begin{array}{l}<7.5 \% \\
\text { Standard }\end{array}$ & $\begin{array}{c}\text { Reference } \\
288(058-1429) \quad 0194\end{array}$ & & & & 063 \\
\hline $\begin{array}{l}7.5 \%-10 \% \\
\text { Standard } \\
\text { Intensive }\end{array}$ & $\begin{array}{c}\text { Reference } \\
2.55(0.67-9.63), 0.166\end{array}$ & 2.33 (0.45-12.14), 0.306 & 0.89 (0.12-6.36), 0.908 & 4.92 (1.07-22.46), $\mathbf{0 . 0 4 0}$ & $1.59(0.75-3.38), 0.222$ \\
\hline $\begin{array}{l}10 \%-15 \% \\
\text { Standard } \\
\text { Intensive }\end{array}$ & $\begin{array}{c}\text { Reference } \\
1.02(0.46-2.28), 0.945\end{array}$ & $3.27(1.30-8.20), \mathbf{0 . 0 1 1}$ & $1.95(0.87-4.38), 0.104$ & $1.61(0.86-3.01), 0.137$ & $1.37(0.75-2.52), 0.310$ \\
\hline
\end{tabular}


TABLE 4: Continued.

\begin{tabular}{lccccc}
\hline ASCVD & Hypotension & Syncope & Bradycardia & Acute renal failure & Electrolyte abnormality \\
\hline $\begin{array}{l}\geq 15 \% \\
\text { Standard }\end{array}$ & $\begin{array}{c}\text { Reference } \\
\text { Intensive }\end{array} 1.17(01.20-2.44), 0.003$ & $1.16(0.84-1.60), 0.364$ & $1.11(0.78-1.57), 0.548$ & $1.67(1.28-2.16),<\mathbf{0 . 0 0 1}$ & $1.28(0.97-1.68), 0.071$ \\
\hline $\begin{array}{l}\text { Total population } \\
\text { Standard }\end{array} \quad \begin{array}{l}\text { Reference } \\
\text { Intensive }\end{array}$ & $1.66(1.22-2.26), \mathbf{0 . 0 0 1}$ & $1.33(0.99-1.78), 0.051$ & $1.18(0.87-1.62), 0.279$ & $1.65(1.31-2.08),<\mathbf{0 . 0 0 1}$ & $1.37(1.09-1.72), \mathbf{0 . 0 0 6}$
\end{tabular}

Data were presented as hazard ratio $(95 \%$ confidence interval), $P$ value. The bold $P$ value data indicates its significance. ASCVD: Atherosclerotic Cardiovascular Disease.

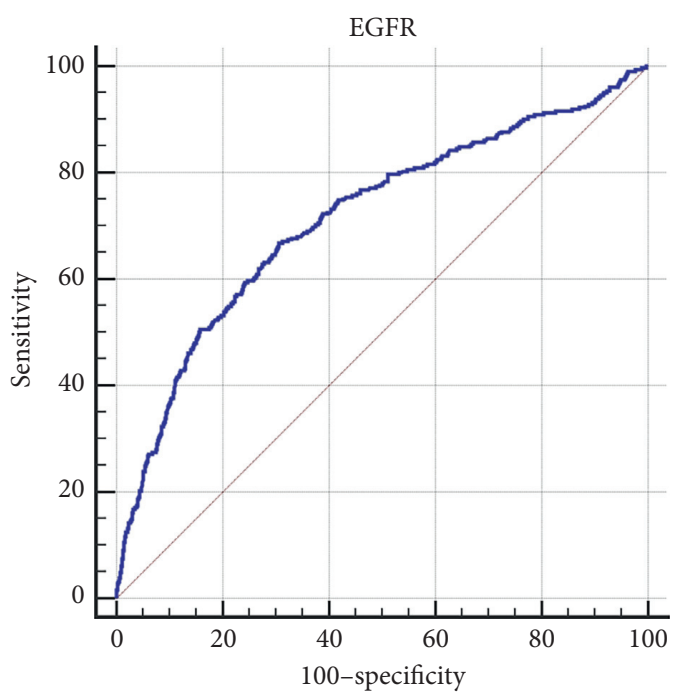

FIGURE 3: ROC curve for prediction of kidney injury based on eGFR is demonstrated. An eGFR below $62 \%$ could be defined as a cut-off point for predicting AKI development with intensive blood pressure reduction.

\section{Discussion}

This study was a post hoc analysis of SPRINT, which investigated the effects of intensive BP treatment on clinical outcomes in patients with different baseline ASCVD risk scores. The results of this study suggested that the intensive control of SBP was able to decrease cardiovascular events in patients with ASCVD risk score of $10 \%$ and above. Furthermore, our study suggested that intensive treatment would be beneficial for primary prevention in patients with ASCVD $\geq 7.5 \%$.

There is still debate as to whether the BP-lowering strategy should be determined on the basis of BP alone. Ogden et al. demonstrated that the absolute benefits of antihypertensive therapy depended not only on the BP level but also on the presence or absence of additional CVD risk factors [15]. The Cardiovascular Health Awareness Program in Canada also reported that management via stratification of risk factors for hypertensive patients reduced CVD mortality in comparison to the usual care [16]. However, many guidelines have not provided recommendations regarding risk-based strategies for hypertension management. This might be due to the lack of enough evidence in this regard. In addition, further studies are needed to determine a proper target of SBP in patients with hypertension who have various comorbidities and CVD risk at baseline $[8,17,18]$.

Zhang et al. compared the effects of intensive BP control and standard treatment among the patients with different baseline Framingham Risk Score (FRS). Their study showed that, in high-risk participants, intensive BP treatment was effective in the risk reduction of the primary outcome. Furthermore, they concluded that the intensive treatment of BP was advantageous in the total population irrespective of the levels of FRS [13]. Although we used a different risk assessment system, our results also showed that the patients with a higher risk of CVD would benefit from intensive BP treatment.

Williamson et al. evaluated the effects of intensive treatment (SBP target $<120 \mathrm{mmHg}$ ) versus standard treatment (SBP target $<140 \mathrm{mmHg}$ ) in patients aged 75 years or older with hypertension, but without diabetes. They found that treating to an SBP target of less than $120 \mathrm{mmHg}$ compared with an SBP target of less than $140 \mathrm{mmHg}$ resulted in significantly lower rates of fatal and nonfatal major cardiovascular events and death from any cause [19]. In agreement with this observation, our analysis suggested that intensive BP control lessened the risk of CVD outcomes in the patients with higher ASCVD risk scores who were older.

In contrast to our study, the ACCORD trial did not identify an SBP target $<120 \mathrm{mmHg}$ advantageous for patients with type $2 \mathrm{DM}$, with the exception of decreased stroke risk [20]. However, in Sprint's study, the patients with DM were excluded.

Attar et al. observed that intensive treatment led to a significant reduction in the primary outcome events in patients with FRS $\geq 10 \%$ [8]. Our results are consistent with this study, as we found that intensive BP control could positively affect the hypertensive patients with ASCVD $\geq 10 \%$.

Although intensive BP treatment can avert cardiovascular events, it can cause some serious adverse health consequences [21-24]. In SPRINT's study, it was shown that the rates of eGFR reduction $\geq 30 \%$ and below $60 \mathrm{ml} / \mathrm{min} / 1.73 \mathrm{~m}^{2}$ and the incidence of SAEs, such as hypotension, syncope, AKI, and electrolyte abnormalities in patients without CKD at baseline were more common in the intensive therapy group [25]. Our study indicated that the incidence of hypotension and AKI was higher among the patients with ASCVD $\geq 15 \%$, which highlighted the fact that the intensive treatment of BP could lead to an increased risk of SAEs.

Similarly, in Accord's study, patients with an achieved SBP target of about $120 \mathrm{mmHg}$ had an increased risk of SAEs 
compared to the patients remaining at an on-treatment SBP of about $133 \mathrm{mmHg}$ [20]. Zhang et al. reported that the risk of SAEs associated with BP treatment significantly increased among the total population, intermediate-risk patients, and high-risk participants [13].

The limitation of our study was that the participants aged under 50 years and the patients with a positive history of stroke, ESRD, diabetes, and congestive HF were excluded. Therefore, the findings of this study could not be generalized to these patients.

\section{Conclusions}

In conclusion, our study showed that, in the patients aged under 75 years without DM, ESRD, or prior stroke who had a 10 -year risk of cardiovascular events above $10 \%$ on the basis of the ASCVD risk score, intensive BP control with an SBP target of less than $120 \mathrm{mmHg}$ significantly reduced the incidence of major cardiovascular events. However, it might be accompanied with an increased risk of SAEs such as hypotension and AKI. It has also been shown that intensive treatment would be beneficial for primary prevention in patients with ASCVD $\geq 7.5 \%$. Totally, our findings suggested that an antihypertensive treatment strategy on the basis of a combination of CVD risk assessment and BP level could bring benefits to the patients.

\section{Abbreviations}

CVD: Cardiovascular disease

SBP: $\quad$ Systolic blood pressure

SPRINT: Systolic Blood Pressure Intervention Trial

ASCVD: Atherosclerotic Cardiovascular Disease

MI: $\quad$ Myocardial infarction

ACS: $\quad$ Acute coronary syndrome

HF: Heart failure

BP: $\quad$ Blood pressure

HR: $\quad$ Hazard ratio

CI: $\quad$ Confidence interval

ESRD: End-stage renal disease

CHF: Congestive heart failure

DBP: Diastolic blood pressure

FRS: $\quad$ Framingham Risk Score

NHLBI: National Heart, Lung, and Blood Institute

CKD: Chronic kidney disease

EGFR: Estimated glomerular filtration rate

DM: Diabetes mellitus

TC: Total cholesterol

HDL-C: High density lipoprotein cholesterol

SADE: Serious adverse events

AKI: Acute renal failure

TG: Triglycerides.

\section{Data Availability}

The SPRINT data were obtained from the National Heart, Lung, and Blood Institute (NHLBI) Data Repository (https://biolincc.nhlbi.nih.gov/studies/sprint/).

\section{Ethical Approval}

This study has been approved by the national ethical committee by an approval number IR.SUMS.MED.REC.1398.377 and conforms to the Declaration of Helsinki.

\section{Consent}

This study has consent from the Shiraz University of Medical Sciences for publication. All participants signed the consent form.

\section{Conflicts of Interest}

The authors declare no conflicts of interest.

\section{Authors' Contributions}

A. Attar and M. S. were involved in conception; A. Attar was responsible for design; A. A. and F. N. carried out data acquisition; M. S., F. N., and A. A. conducted data interpretation; M. S., F. N., and A. A. analysed data. All authors drafted and revised the manuscript and finally approved the manuscript

\section{Acknowledgments}

The authors would like to thank the Center for Development of Clinical Research of Nemazee Hospital and Dr. Nasrin Shokrpour for editorial assistance. This project has been performed under Grant no. 97-01-01-17712 from vicechancellor of research of Shiraz University of Medical Sciences, Shiraz, Iran.

\section{References}

[1] H. B. Bosworth, R. M. Bartash, M. K. Olsen, and D. C. Steffens, "The association of psychosocial factors and depression with hypertension among older adults," International Journal of Geriatric Psychiatry, vol. 18, no. 12, pp. 1142-1148, 2003.

[2] W. S. Aronow, "Hypertension guidelines," Hypertension, vol. 58, no. 3, pp. 347-348, 2011.

[3] C. Johnson, "Dietary sodium and blood pressure in older adults," Californian Journal of Health Promotion, vol. 4, 2006.

[4] M. Brunström, N. Ng, J. Dahlström et al., "Association of physician education and feedback on hypertension management with patient blood pressure and hypertension control," JAMA Network Open, vol. 3, no. 1, e1918625 pages, 2020.

[5] S. R. Group, "A randomized trial of intensive versus standard blood-pressure control," New England Journal of Medicine, vol. 373, no. 22, pp. 2103-2116, 2015.

[6] A. A. Leung, K. Nerenberg, S. S. Daskalopoulou et al., "Hypertension Canada's 2016 Canadian hypertension education Program guidelines for blood pressure measurement, diagnosis, assessment of risk, prevention, and treatment of hypertension," The Canadian Journal of Cardiology, vol. 32, no. 5, pp. 569-588, 2016.

[7] T. C. Lee, R. B. Cavalcanti, E. G. McDonald, L. Pilote, and J. M. Brophy, "Diastolic hypotension may attenuate benefits from intensive systolic targets: secondary analysis of a 
randomized controlled trial," The American Journal of Medicine, vol. 131, no. 10, pp. 1228-1233.e1, 2018.

[8] A. Attar, M. Sayadi, and M. Jannati, "Effect of intensive blood pressure lowering on cardiovascular outcomes based on cardiovascular risk: a secondary analysis of the SPRINT trial," European Journal of Preventive Cardiology, vol. 26, no. 3, pp. 238-245, 2019.

[9] K. N. Karmali, D. M. Lloyd-Jones, J. Van Der Leeuw et al., "Blood pressure-lowering treatment strategies based on cardiovascular risk versus blood pressure: a meta-analysis of individual participant data," PLoS Medicine, vol. 15, no. 3, Article ID e1002538, 2018.

[10] S. E. Berman, L. A. Rivera-Rivera, L. R. Clark et al., "Intracranial arterial four-dimensional flow is associated with metrics of brain health and Alzheimer's disease," Alzheimer's \& Dementia: Diagnosis, Assessment \& Disease Monitoring, vol. 1, no. 4, pp. 420-428, 2015.

[11] D. C. Goff, D. M. Lloyd-Jones, G. Bennett et al., "2013 ACC/ AHA guideline on the assessment of cardiovascular risk," Circulation, vol. 129, no. 25 Suppl 2, pp. S49-S73, 2014.

[12] S. Beddhu, G. M. Chertow, T. Greene et al., "Effects of intensive systolic blood pressure lowering on cardiovascular events and mortality in patients with type 2 diabetes mellitus on standard glycemic control and in those without diabetes mellitus: reconciling results from ACCORD BP and SPRINT," Journal of American Heart Association, vol. 7, no. 18, Article ID e009326, 2018.

[13] L. Zhang, X. Sun, L. Liao et al., "Effectiveness of blood pressure-lowering treatment by the levels of baseline Framingham risk score: a post hoc analysis of the systolic blood pressure intervention trial (SPRINT)," The Journal of Clinical Hypertension, vol. 21, no. 12, pp. 1813-820, 2019.

[14] W. T. Ambrosius, K. M. Sink, C. G. Foy et al., "The design and rationale of a multicenter clinical trial comparing two strategies for control of systolic blood pressure: the systolic blood pressure intervention trial (SPRINT)," Clinical Trials: Journal of the Society for Clinical Trials, vol. 11, no. 5, pp. 532-546, 2014.

[15] L. G. Ogden, J. He, E. Lydick, and P. K. Whelton, "Long-term absolute benefit of lowering blood pressure in hypertensive patients according to the JNC VI risk stratification," Hypertension, vol. 35, no. 2, pp. 539-543, 2000.

[16] E. Y. T. Yu, E. Y. F. Wan, C. K. H. Wong et al., "Effects of risk assessment and management programme for hypertension on clinical outcomes and cardiovascular disease risks after 12 months," Journal of Hypertension, vol. 35, no. 3, pp. 627-636, 2017.

[17] T. A. Gaziano, K. Steyn, D. J. Cohen, M. C. Weinstein, and L. H. Opie, "Cost-effectiveness analysis of hypertension guidelines in South Africa," Circulation, vol. 112, no. 23, pp. 3569-3576, 2005.

[18] A. Zanchetti, G. Grassi, and G. Mancia, "When should antihypertensive drug treatment be initiated and to what levels should systolic blood pressure be lowered? a critical reappraisal," Journal of Hypertension, vol. 27, no. 5, pp. 923-934, 2009.

[19] S. Kulenthiran, S. Ewen, M. Böhm, and F. Mahfoud, "Hypertension up to date: SPRINT to SPYRAL," Clinical Research in Cardiology, vol. 106, no. 7, pp. 475-484, 2017.

[20] J. D. Williamson, M. A. Supiano, W. B. Applegate et al., "Intensive vs standard blood pressure control and cardiovascular disease outcomes in adults aged $\geq 75$ years," JAMA, vol. 315, no. 24, pp. 2673-2682, 2016.
[21] S. Basu, J. B. Sussman, J. Rigdon et al., "Benefit and harm of intensive blood pressure treatment: derivation and validation of risk models using data from the SPRINT and ACCORD trials," PLoS Medicine, vol. 14, no. 10, Article ID e1002410, 2017.

[22] A. Attar, A. sadeghi, F. Amirmoezzi, and K. Aghasadesghi, "Low dose spironolactone monotherapy in the management of stage I essential hypertension: a pilot randomized, doubleblind, placebo-controlled trial," Acta Cardiologica Sinica, vol. 54, no. 1, pp. 59-65, 2018.

[23] M. Khosravi Maharlooei, A. Attar, A. Goran et al., "Hydatid cyst of ovary: a case report," Iranian Journal of Medical Sciences, vol. 34, no. 1, pp. 76-79, 2009.

[24] M. Khosravi-Maharlooei, M. Jaberipour, A. H. Tashnizi et al., "Expression pattern of alternative splicing variants of human telomerase reverse transcriptase (hTERT) in cancer cell lines was not associated with the origin of the cells," International journal of Molecular and Cellular Medicine, vol. 4, no. 2, pp. 109-119, 2015.

[25] V. Perkovic and A. Rodgers, "Redefining blood-pressure targets-SPRINT starts the marathon," New England Journal of Medicine, vol. 373, no. 22, pp. 2175-2178, 2015. 